\title{
REFERENCE MODEL DECOMPOSITION IN DIRECT ADAPTIVE CONTROL
}

\author{
H. BUTLER AND G. HONDERD \\ Department of Electrical Engineering, Control Laboratory, Delft University of Technology, PO Box 5031, \\ NL-2600 GA Delft, The Netherlands \\ AND \\ J. VAN AMERONGEN \\ Department of Electrical Engineering, Control, Systems and Computer Engineering Laboratory, \\ University of Twente, PO Box 2I7, NL-7500 AE Enschede, The Netherlands
}

\begin{abstract}
SUMMARY
This paper introduces the method of 'reference model decomposition' as a way to improve the robustness of model reference adaptive control systems (MRACs) with respect to unmodelled dynamics with a known structure. Such unmodelled dynamics occur when some of the nominal plant dynamics are purposely neglected in the controller design with the aim of keeping the controller order low. One of the effects of such 'undermodelling' of the controller is a violation of the perfect model-matching condition of the primary controller. The decomposition can be seen as a way to adjust the reference model output (and hence the control goal) to the actual model-matching capabilities. It is shown that the decomposition alleviates the negative effects unmodelled dynamics have on the error equation. Simulation examples illustrate the decomposition design steps and show the obtained improvements.
\end{abstract}

KEY WORDS Model reference adaptive control Unmodelled dynamics Robustness Reference model adjustment

\section{INTRODUCTION}

The problem of the robustness of MRAC systems with respect to bounded and state-dependent disturbances has gained much attention in the literature. ${ }^{1-7}$ The problem of bounded disturbances has led to several modifications of the adaptive law, such as a dead zone, ${ }^{8}$ restriction of the parameters to a known region ${ }^{9}$ and shifting the adaptation pole from the origin to the negative real axis in the $s$-plane. This shift can be permanent or dependent on the actual output error magnitude. ${ }^{6,7}$ All modifications provide stability of the adaptive system provided that the modification parameters are properly tuned to the disturbance magnitude.

On the other hand, research has focused on the persistently exciting (PE) properties of signals in the adaptive loop as a way to guarantee stability. Narendra and Annaswamy ${ }^{7}$ and later Lee and Narendra ${ }^{5}$ proved that a sufficiently large degree of persistent excitation provides stability of the adaptive system in the presence of bounded disturbances.

The problem of unmodelled dynamics is fundamentally different from that of bounded disturbances in that no upper bound on the disturbance can be guaranteed and the error

This paper was recommended for publication by editor $R$. Bitmead 
equation's strictly positive real (SPR) property is violated. Usually, the unmodelled dynamics considered lie in a frequency range outside the plant bandwidth because especially in the highfrequency range structural knowledge of the plant dynamics is missing. If the unmodelled dynamics have only a relatively small effect on the process output, simple adaptive law modifications are sufficient to deal with them. ${ }^{6}$

In this paper a somewhat different problem setting is considered. Here the unmodelled dynamics are assumed to be in the same frequency range as the plant bandwidth. In addition, structural knowledge of the unmodelled dynamics and an indication of the relevant parameter values are assumed to be present. Such a problem setting occurs when the designer wants to keep the number of adjustable parameters low and hence the controller has too low an order to achieve perfect model matching. This problem setting implies a division of the plant dynamics into three classes: the nominal part for which the controller is designed; the structured unmodelled part which is known to exist but disregarded to keep the controller order low; and the unmodelled part (usually in the high-frequency range) which is disregarded altogether. In the literature most attention is focused on the last class, while this paper considers the second class. The new method of reference model decomposition is presented as a way of dealing with these 'structured unmodelled dynamics'.

One may argue that structured unmodelled dynamics are no unmodelled dynamics at all and that a full-order controller can be used. However, practical observations lead to the conclusion that a minimum number of parameters to be adjusted is favourable at all times. A larger number of adjustable parameters needs more computer time, usually induces a slower convergence, and the algorithm is generally more sensitive to non-linearities. This is the reason why reports on practical MRAC applications normally use relatively simple algorithms. ${ }^{10-16}$

The decomposition method to be described can be regarded as a way to include the effect of the unmodelled dynamics in the reference model output. This inclusion is not exact but is modified such that the model output is still a desired output. The difference between the new decomposed model output and the process output is in this way made less sensitive to the unmodelled dynamics, and hence no parameter updating is done as a reaction to these dynamics. Alternatively stated, the reference model output is modified such that it is corrected for the limited model-matching capabilities of the (low-order) primary controller. Hence the decomposition method can be regarded as a way of finding a compromise between the limited model-matching capabilities on the one hand and the resulting closed-loop behaviour on the other hand. In addition to the improved stability properties, an extra advantage appears to be a reduced control effort.

This paper is organized as follows. Section 2 describes the MRAC scheme to be used throughout the paper and analyses the effect of unmodelled dynamics on the system. Section 3 introduces the concept of reference model decomposition, shows its positive effects on the error equations and considers the choice of the decomposition parameters. Section 4 presents two examples illustrating the decomposition method and shows the obtained results. Section 5 summarizes the decomposition design steps and, finally, Section 6 gives some conclusions regarding the decomposition method.

\section{THE ADAPTIVE CONTROLLER AND UNMODELLED DYNAMICS}

\subsection{The adaptive controller}

Although the decomposition principle is applicable to any MRAC structure, in this paper the method using an error-augmenting signal will serve as a framework (see, among others, 


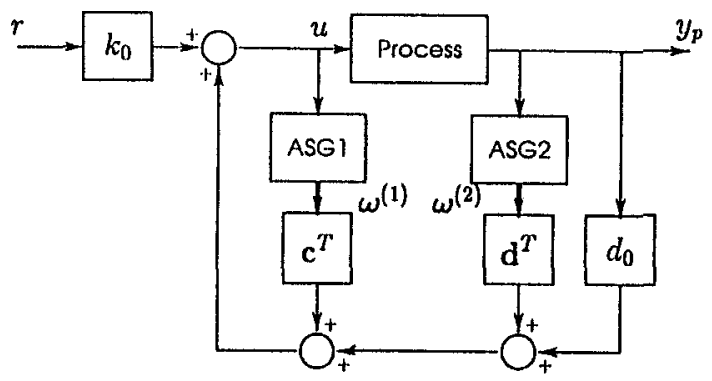

Figure 1. Basic controller structure

Reference 17). The basic controller structure is depicted in Figure 1, showing that two 'auxiliary signal generators' (ASGs) produce signal vectors $\omega^{(1)}$ and $\omega^{(2)}$, both of order $n-1$. These signal vectors are multiplied by parameter vectors $c^{T}$ and $\mathbf{d}^{\mathrm{T}}$ and the result is fed back to the plant input. The following notation will be used for the plant transfer function:

$$
W_{\mathrm{p}}=k_{\mathrm{p}} \frac{Z_{\mathrm{p}}}{R_{\mathrm{p}}}, \quad Z_{\mathrm{p}}=s^{m}+\sum_{i=0}^{m-1} b_{i} s^{i}, \quad R_{\mathrm{p}}=s^{n}+\sum_{i=0}^{n-1} a_{i} s^{i}
$$

and the transfer function of the reference model:

$$
W_{\mathrm{m}}=k_{\mathrm{m}} \frac{Z_{\mathrm{m}}}{R_{\mathrm{m}}}
$$

The relative degree of the reference model transfer function is assumed to be $n-m$. Further,

$$
\begin{aligned}
\boldsymbol{\omega}^{\mathrm{T}}=\left(r, \boldsymbol{\omega}^{(1) \mathrm{T}}, y_{\mathrm{p}}, \boldsymbol{\omega}^{(2) \mathrm{T}}\right): & \text { the signal vector } \\
\boldsymbol{\theta}^{\mathrm{T}}=\left(k_{0}, \mathbf{c}^{\mathrm{T}}, d_{0}, \mathbf{d}^{\mathrm{T}}\right): & \text { the controller parameter vector } \\
\boldsymbol{\theta}^{*}: & \text { the correct controller parameter vector } \\
\boldsymbol{\phi}=\boldsymbol{\theta}-\boldsymbol{\theta}^{*}: & \text { the parameter error vector }
\end{aligned}
$$

$W_{\mathrm{p}}$ must be minimum phase. As described by Narendra and Annaswamy, ${ }^{17}$ the auxiliary signal generators take the form of linear filters of order $n-1$. The transfer function of the signal generators, including the parameter vectors $c^{T}$ and $d^{T}$, can be written as

$$
\begin{aligned}
\text { ASGl: } & \frac{C}{Q}, \text { with } C=\underbrace{c_{n-1} s^{n-2}+\cdots+c_{2} s+c_{1}}_{[n-2]} \\
Q & =\underbrace{s^{n-1}+\cdots+q_{1} s+q_{0}}_{[n-1]}
\end{aligned}
$$

ASG2: $\frac{D}{Q}, \quad$ with $D=\underbrace{d_{n-1} s^{n-2}+\cdots+d_{2} s+d_{1}}_{[n-2]}$

The ASG denominator polynomial $Q$ must include the reference model zeros in order to satisfy the perfect model-matching condition.

By changing the transfer function of ASG2 to $s D / Q$, this signal generator functions as a state-reconstructing element and $\omega^{(2)}$ consists of an estimate of the internal plant state vector. Extensive simulations have shown that by this change convergence is improved, especially if the plant has badly damped poles. The perfect model-matching condition is still satisfied and 
the adaptive laws remain unaltered. A similar change of ASG1 is not useful and would give implementation problems due to an occurring algebraic loop. The modified ASG2 is used in all experiments in this paper. The output error $e_{1}=y_{\mathrm{p}}-y_{\mathrm{m}}$ can be derived to be ${ }^{17}$

$$
e_{1}=\frac{k_{\mathrm{p}}}{k_{\mathrm{m}}} W_{\mathrm{m}}\left(\phi^{\mathrm{T}} \omega\right)
$$

In the following we will assume $k_{\mathrm{p}}=k_{\mathrm{m}}=1$. The plant output can be written as

$$
y_{\mathrm{p}}=W_{\mathrm{m}}\left(r+\phi^{\mathrm{T}} \omega\right)
$$

If $W_{\mathrm{m}}$ is SPR, the well-known integral adaptive law can be applied directly:

$$
\dot{\theta}=-\Gamma \omega e_{1}, \quad \Gamma>0
$$

In this equation $\Gamma$ is the adaptation gain matrix. If $W_{\mathrm{m}}$ is not SPR, an error-augmenting network must be used, adding an extra signal to the output error $e_{1}$ :

$$
\begin{aligned}
\varepsilon & =e_{1}+W_{\mathrm{m}} L\left(\theta L^{-1}-L^{-1} \theta\right)^{\mathrm{T}} \omega \\
& =e_{1}+W_{\mathrm{m}} L\left(\phi L^{-1}-L^{-1} \phi\right)^{\mathrm{T}} \omega \\
& =W_{\mathrm{m}}\left(\phi^{\mathrm{T}} \omega\right)+W_{\mathrm{m}} L\left(\phi L^{-1}-L^{-1} \phi\right)^{\mathrm{T}} \omega \\
& =W_{\mathrm{m}} L\left(\phi^{\mathrm{T}} L^{-1} \omega\right) \\
& =W_{\mathrm{m}} L\left(\phi^{\mathrm{T}} \xi\right)
\end{aligned}
$$

The rational transfer function $L$ must be chosen such that $W_{\mathrm{m}} L$ is SPR. The filters $L^{-1}$ make the error equation SPR and generate the filtered signal vector $\xi$ to be used in the adaptation from $\omega$ :

$$
\dot{\boldsymbol{\theta}}=-\Gamma\left(L^{-1} \omega\right) \varepsilon=-\Gamma \xi \varepsilon
$$

\subsection{Unmodelled dynamics}

The main interest in the effects of unmodelled plant dynamics in model reference adaptive control systems was started by Rohrs et al. ${ }^{18}$ Rohrs' examples showed that even if the unmodelled dynamics play a role only in a frequency area outside the nominal plant bandwidth, and the poles of the unmodelled part are properly damped, instability may occur. His examples aroused a large interest in the mechanisms playing a role in this instability. It was found that two mechanisms play a vital role. ${ }^{19,20}$

First, if the reference signal $r$ does not have sufficient richness, ambiguity in the controller parameters remains. A small amount of measurement noise may then induce parameter drift, resulting in instability due to the presence of unmodelled dynamics in the process. This mechanism is called slow drift instability. ${ }^{20}$

Secondly, a reference signal which is exciting in the frequency area in which the unmodelled dynamics play a role causes the adaptation to attempt model matching in this frequency area. However, the primary controller is not equipped to achieve model matching in this frequency range and therefore instability may result. This mechanism is especially of interest in this paper, because here the frequency range in which the unmodelled dynamics are active is in the same range as the nominal plant bandwidth.

The unmodelled dynamics in the process will be described as a transfer function $\widetilde{W}_{\mathrm{p}}$ which is added to the original process transfer $W_{\mathrm{p}}:{ }^{6}$

$$
\bar{W}_{\mathrm{p}}=W_{\mathrm{p}}+\widetilde{W}_{\mathrm{p}}
$$


Here $\bar{W}_{\mathrm{p}}$ is the complete process transfer function consisting of the nominal part $W_{\mathrm{p}}$ and an unmodelled part $\widetilde{W}_{\mathrm{p}}$. This process is controlled by a MRAC system which was designed for only $W_{\mathrm{p}}$, and so a parameter vector $\theta^{*}$ in combination with a signal vector $\omega^{*}$ makes the output of $W_{\mathrm{p}}$ equal to the reference model output:

$$
W_{\mathrm{p}}\left(\theta^{* \mathrm{~T}} \omega^{*}\right)=W_{\mathrm{m}} r
$$

It is assumed that the true parameter vector $\theta^{*}$ in the undisturbed case still stabilizes the system if there are unmodelled dynamics. Note that the signal vector then is not equal to $\omega^{*}$ but will be denoted $\omega$. Of course, the process output in this case does not equal the reference model output $y_{\mathrm{m}}=W_{\mathrm{m}} r$. A fictitious reference model $\bar{W}_{\mathrm{m}}$ is defined as the closed-loop response if the primary controller were equipped with the originally exact parameter vector $\theta^{*}::^{6}$

$$
\bar{W}_{\mathrm{m}} r=\bar{W}_{\mathrm{p}}\left(\theta^{* \mathrm{~T}} \boldsymbol{\omega}\right)
$$

Because the unmodelled dynamics in the process are not known, the fictitious reference model transfer $\bar{W}_{\mathrm{m}}$ is unknown too. The main reason for defining $\bar{W}_{\mathrm{m}} r$ as in (4) is that the plant output can be written as ${ }^{6}$

$$
y_{\mathrm{p}}=\bar{W}_{\mathrm{m}}\left(r+\phi^{\mathrm{T}} \omega\right)
$$

If $\phi=0$ and so $\theta=\theta^{*}$, (4) reappears. To analyse the effect of the unmodelled dynamics on the error equation, two situations can be distinguished.

If $W_{\mathrm{m}}$ is SPR, the output error $e_{1}=y_{\mathrm{p}}-y_{\mathrm{m}}$ can be used directly in the adaptation in the nominal case. The error equation becomes

$$
\begin{aligned}
e_{1} & =y_{\mathrm{p}}-y_{\mathrm{m}} \\
& =\bar{W}_{\mathrm{m}}\left(r+\phi^{\mathrm{T}} \omega\right)-W_{\mathrm{m}} r \\
& =\bar{W}_{\mathrm{m}}\left(\phi^{\mathrm{T}} \omega\right)+\widetilde{W}_{\mathrm{m}} r \\
& =\bar{W}_{\mathrm{m}}\left(\phi^{\mathrm{T}} \omega\right)+\nu
\end{aligned}
$$

In (6) an output disturbance $\widetilde{W}_{\mathrm{m}} r$ on the output error appears, with $\widetilde{W}_{\mathrm{m}}=\bar{W}_{\mathrm{m}}-W_{\mathrm{m}}$. This disturbance can be written in the alternative form

$$
\begin{aligned}
\bar{W}_{\mathrm{p}}\left(\theta^{* \mathrm{~T}} \omega\right) & =\bar{W}_{\mathrm{m}} r \\
W_{\mathrm{p}}\left(\theta^{* \mathrm{~T}} \omega\right)+\widetilde{W}_{\mathrm{p}}\left(\theta^{* \mathrm{~T}} \omega\right) & =W_{\mathrm{m}} r+\widetilde{W}_{\mathrm{m}} r \\
W_{\mathrm{p}}\left(\theta^{* \mathrm{~T}}\left(\omega-\omega^{*}\right)\right)+W_{\mathrm{p}}\left(\theta^{* \mathrm{~T}} \omega^{*}\right)+\bar{W}_{\mathrm{p}}\left(\theta^{* \mathrm{~T}} \omega\right) & =W_{\mathrm{m}} r+\widetilde{W}_{\mathrm{m}} r
\end{aligned}
$$

Using (3), this yields

$$
\widetilde{W}_{\mathrm{m}} r=W_{\mathrm{p}}\left(\theta^{* \mathrm{~T}}\left(\boldsymbol{\omega}-\boldsymbol{\omega}^{*}\right)\right)+\widetilde{W}_{\mathrm{p}}\left(\theta^{* \mathrm{~T}} \boldsymbol{\omega}\right)
$$

Equation (7) shows that the disturbance on the output error (which is equal to the effect of the unmodelled dynamics on $y_{\mathrm{p}}$ for $\theta=\theta^{*}$ ) consists of two parts. Suppose for a moment that $\phi=0$, so that $e_{1}$ is equal to the output disturbance only and is not influenced by the adaptation error (the first term in (6)). In this case the output error $e_{1}$ is equal to $\widetilde{W}_{\mathrm{m}} r$ as in (7). The first term in (7) is the output of the nominal process, with as input the product of the parameter vector and the difference between the actual signal vector $\omega$ and the originally perfect signal vector $\omega^{*}$. Thus the first term consists of the output of the nominal plant, with as input a signal which is completely due to the penetration of the unmodelled dynamics in $\omega$. The second term in (7) corresponds to the output of the unmodelled part of the plant, with as input the actual process input $u=\theta^{* \mathrm{~T}} \omega$.

If $W_{\mathrm{m}}$ is not SPR, an error-augmenting network is needed and the signal vector $\omega$ changes 
to $\xi=L^{-1} \omega$. The augmented error becomes

$$
\begin{aligned}
\varepsilon & =e_{1}-W_{\mathrm{m}} L\left(L^{-1} \phi-\phi L^{-1}\right)^{\mathrm{T}} \boldsymbol{\omega} \\
& =\bar{W}_{\mathrm{m}}\left(\phi^{\mathrm{T}} \omega\right)+\bar{W}_{\mathrm{m}} r-W_{\mathrm{m}}\left(\phi^{\mathrm{T}} \omega\right)+W_{\mathrm{m}} L\left(\phi^{\mathrm{T}}\left(L^{-1} \omega\right)\right) \\
& =\bar{W}_{\mathrm{m}} L\left(\phi^{\mathrm{T}} L^{-1} \omega\right)+\widetilde{W}_{\mathrm{m}} L\left(L^{-1} \phi-\phi L^{-1}\right)^{\mathrm{T}} \boldsymbol{\omega}+\bar{W}_{\mathrm{m}} r \\
& =\bar{W}_{\mathrm{m}} L\left(\phi^{\mathrm{T}} \xi\right)+\nu^{\prime}
\end{aligned}
$$

Now a disturbance $\nu^{\prime}$ arises which is different from $\nu$ owing to the error-augmenting signal which is only equipped for $W_{\mathrm{m}}$ instead of $\bar{W}_{\mathrm{m}}$. Comparing the error equations (6) and (8) with the original equations (1) and (2), two effects of the unmodelled dynamics can be observed.

First, it is seen that an output disturbance $\nu$ or $\nu^{\prime}$ is present. If $W_{\mathrm{m}}$ is SPR, this disturbance equals $\nu=\widetilde{W}_{\mathrm{m}} r$, and because it was assumed that the original exact parameter vector $\theta^{*}$ stabilizes the overall system, $\nu$ is bounded. If $W_{\mathrm{m}}$ is not SPR, the disturbance $\nu^{\prime}$ includes a term due to an incorrect error-augmenting signal and boundedness is not automatically guaranteed. All modifications for dealing with external disturbances, such as the inclusion of a dead zone or shifting the adaptation pole, can be used to minimize the effect of $\nu$. More formally, if there is an upper bound $\nu_{0}$ on the disturbance $\nu$ or $\nu^{\prime}$ that satisfies

$$
\nu_{0} \leqslant \beta\left(1+\xi^{\mathrm{T}} \xi\right)^{1 / 2}
$$

for a sufficiently small $\beta$, these adaptive law modifications guarantee stability. ${ }^{6}$ Thus, if the disturbance due to unmodelled dynamics is small compared to the signal vector, stability can be guaranteed by using a standard adaptive law modification.

Secondly, the linear error transfer function has changed from $W_{\mathrm{m}}$ to $\bar{W}_{\mathrm{m}}$ or from $W_{\mathrm{m}} L$ to $\bar{W}_{\mathrm{m}} L$. Because $\bar{W}_{\mathrm{m}}$ and $\bar{W}_{\mathrm{m}} L$ include an unknown part due to the unmodelled process dynamics, the SPR property of the error equation is endangered. This problem can be dealt with by using the theory of averaging. ${ }^{3,20}$ Under the assumption of a small adaptation gain, the averaging technique shows that the strictly positive real property on the linear part of the error equation may be violated as long as the error transfer has a positive real part for the 'most important' frequencies in the signal vector. Roughly stated, this averaging result says that the larger the frequency range over which $\bar{W}_{\mathrm{m}}(\mathrm{j} \omega)$ or $\bar{W}_{\mathrm{m}} L(\mathrm{j} \omega)$ has a positive real part, the better are the stability properties of the adaptive system.

To summarize, the presence of unmodelled dynamics in the process results in two types of disturbances in the error equation. An output disturbance occurs which effectively tells that the perfect model-matching condition is violated. In addition, the SPR property of the linear part is endangered, which may be harmless if $\operatorname{Re}\left[\bar{W}_{\mathrm{m}}(\mathrm{j} \omega)\right]$ or $\operatorname{Re}\left[\bar{W}_{\mathrm{m}} L(\mathrm{j} \omega)\right]$ is positive for the most important frequencies in $\xi$. Note that decreasing the adaptive gains leads to a larger separation of the adaptation dynamics from the process dynamics, in turn resulting in a wider application of the averaging principle.

\section{REFERENCE MODEL DECOMPOSITION}

\subsection{Basic decomposition idea}

In applying the decomposition, a system $H$ to be decomposed is first separated into a nominal part $H_{\mathrm{n}}$ and an unmodelled part $H_{\mathrm{u}}$ :

$$
H=H_{\mathrm{n}} H_{\mathrm{u}}
$$

Next, $H_{\mathrm{u}}$ is decomposed into three polynomials $T_{1}, T_{2}$ and $N$ as shown in Figure 2. The 


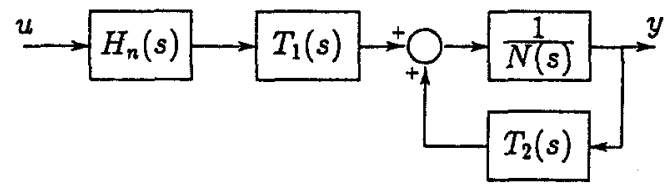

Figure 2. Decomposition of $H(s)$ into $T_{1}(s), T_{2}(s)$ and $N(s)$

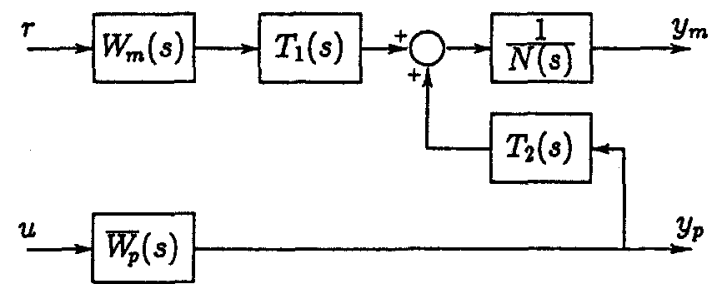

Figure 3. Use of the decomposition principle in MRAC

decomposition polynomials must be chosen such that

$$
\frac{T_{1}}{N-T_{2}}=H_{\mathrm{u}}
$$

This leads to the observation that $T_{1}$ and $N-T_{2}$ are determined by $H_{\mathrm{u}}$, but $N$ may be chosen freely as long as the order of $N$ is equal to the order of the denominator polynomial of $H_{\mathrm{u}}$. The decomposition described is always possible.

If $H$ is a reference model, the output of $H$ is used to calculate an error signal between the process and model outputs. The decomposition effect is then achieved by feeding back not $y_{\mathrm{m}}$ via $T_{2}$, but feeding back $y_{\mathrm{p}}$ as shown in Figure 3. Here the nominal transfer $H_{\mathrm{n}}$ consists of the original reference model $W_{\mathrm{m}}$, and the decomposition polynomials $T_{1}, T_{2}$ and $N$ perform a correction of the reference model output $y_{\mathrm{m}}$. The decomposition polynomials are not considered a part of the reference model, but an addition to the existing structure. The polynomials $T_{1}, T_{2}$ and $N$ together form the decomposition model. The correction polynomial $T_{2}$ is meant to make the reference model output have a similar 'unmodelled' part as the process output, which becomes clear by considering that the states in $1 / N$ should resemble the 'unmodelled' states in the process. For an exact decomposition the polynomials $T_{1}$ and $N-T_{2}$ should be the same as those that would appear in a process decomposition, which is, however, not exactly available. $T_{2}$ corrects the states in $1 / N$ for mismatch between the decomposition parameters and the actual process parameters.

The method of decomposition can be regarded as a special form of model updating ${ }^{21}$ but, however, takes place continuously instead of intermittently. Decomposition also has similarities with series-parallel MRAC.

\subsection{Effects of decomposition on the error equation}

General effects. In the decomposition analysis also, $\theta^{*}$ is defined as the parameter vector for which $W_{\mathrm{p}}\left(\theta^{* \mathrm{~T}} \omega^{*}\right)=W_{\mathrm{m}} r$. For this analysis, only a strictly positive real $W_{\mathrm{m}}$ is considered. 
Analysing Figure 3, the output error can be written as

$$
\begin{aligned}
e_{1}=y_{\mathrm{p}}-y_{\mathrm{m}} & =y_{\mathrm{p}}-\frac{T_{1}}{N} W_{\mathrm{m}} r-\frac{T_{2}}{N} y_{\mathrm{p}} \\
& =\left(\frac{N-T_{2}}{N}\right) y_{\mathrm{p}}-\frac{T_{1}}{N} W_{\mathrm{m}} r
\end{aligned}
$$

Using (5), this yields

$$
\begin{aligned}
e_{1} & =\left(\frac{N-T_{2}}{N}\right) \bar{W}_{\mathrm{m}}\left(r+\phi^{\mathrm{T}} \omega\right)-\frac{T_{1}}{N} W_{\mathrm{m}} r \\
& =\left(\frac{N-T_{2}}{N}\right) \bar{W}_{\mathrm{m}}\left(\phi^{\mathrm{T}} \omega\right)+\left(\frac{N-T_{2}}{N}\right) \bar{W}_{\mathrm{m}} r-\frac{T_{1}}{N} W_{\mathrm{m}} r
\end{aligned}
$$

Next, $y_{\mathrm{m}}^{*}$ is denoted the output of the reference model corrected by the decomposition, for $\theta=\theta^{*}$ :

$$
\begin{aligned}
y_{\mathrm{m}}^{*} & =\frac{T_{1}}{N} W_{\mathrm{m}} r+\frac{T_{2}}{N} y_{\mathrm{p}}^{*} \\
& =\frac{T_{1}}{N} W_{\mathrm{m}} r+\frac{T_{2}}{N} \bar{W}_{\mathrm{p}}\left(\theta^{* \mathrm{~T}} \omega\right)
\end{aligned}
$$

Using this definition and (4), it easily follows that the error equation can be written as

$$
e_{1}=\left(\frac{N-T_{2}}{N}\right) \bar{W}_{\mathrm{m}}\left(\phi^{\mathrm{T}} \omega\right)+\bar{W}_{\mathrm{m}} r-y_{\mathrm{m}}^{*}
$$

Equation (11) shows two differences with regard to the original error equation (6).

First, the error transfer $\bar{W}_{\mathrm{m}}$ has changed to $\left[\left(N-T_{2}\right) / N\right] \bar{W}_{\mathrm{m}}$. By choosing the decomposition parameters (in particular the polynomial $N$ ), the linear part of the error equation can be influenced, making it possible to make this part more positive real. In other words, the frequency range over which $\operatorname{Re}\left[\bar{W}_{\mathrm{m}}(\mathrm{j} \omega)\right]>0$ can be increased. This is advantageous because the averaging result mentioned in Section 2.2. directly indicates better stability properties.

Secondly, the output disturbance has changed from $\nu=\left(\bar{W}_{\mathrm{m}}-W_{\mathrm{m}}\right) r$ to $\nu=\bar{W}_{\mathrm{m}} r-y_{\mathrm{m}}^{*}$. By the assumptions made, this latter disturbance is bounded. A further analysis of this disturbance enhances the understanding of the decomposition effects.

Effect on the output disturbance. Combining equations (4) and (10) gives

$$
\begin{aligned}
\bar{W}_{\mathrm{m}} r-y_{\mathrm{m}}^{*} & =\bar{W}_{\mathrm{p}}\left(\theta^{* \mathrm{~T}} \omega\right)-\left(\frac{T_{1}}{N} W_{\mathrm{m}} r+\frac{T_{2}}{N} \bar{W}_{\mathrm{p}}\left(\theta^{* \mathrm{~T}} \omega\right)\right) \\
& =\left(\frac{N-T_{2}}{N}\right) \bar{W}_{\mathrm{p}}\left(\theta^{* \mathrm{~T}} \omega\right)-\frac{T_{1}}{N} W_{\mathrm{m}} r \\
& =\left(\frac{N-T_{2}}{N}\right) \bar{W}_{\mathrm{p}}\left(\theta^{* \mathrm{~T}} \omega\right)-\frac{T_{1}}{N} W_{\mathrm{p}}\left(\theta^{* \mathrm{~T}} \omega^{*}\right) \\
& =\left(\frac{N-T_{2}}{N}\right) \bar{W}_{\mathrm{p}}\left(\theta^{* \mathrm{~T}} \omega\right)-\frac{T_{1}}{N} W_{\mathrm{p}}\left(\theta^{* \mathrm{~T}} \omega\right)+\frac{T_{1}}{N} W_{\mathrm{p}}\left(\theta^{* \mathrm{~T}}\left(\omega-\omega^{*}\right)\right)
\end{aligned}
$$


Using (7), this becomes

$$
\bar{W}_{\mathrm{m}} r-y_{\mathrm{m}}^{*}=\left(\frac{N-T_{2}}{N}\right) \bar{W}_{\mathrm{p}}\left(\theta^{* \mathrm{~T}} \omega\right)-\frac{T_{1}}{N} W_{\mathrm{p}}\left(\theta^{* \mathrm{~T}} \omega\right)+\frac{T_{1}}{N}\left(\widetilde{W}_{\mathrm{m}} r-\widetilde{W}_{\mathrm{p}}\left(\theta^{* \mathrm{~T}} \omega\right)\right)
$$

Considering the link between the original form of describing the unmodelled dynamics by $\widetilde{W}_{\mathrm{p}}$ and' a process decomposition in the form of polynomials $T_{\mathrm{p} 1}, T_{\mathrm{p} 2}$ and $N_{\mathrm{p}}$, i.e.

$$
\begin{aligned}
\bar{W}_{\mathrm{p}} & =W_{\mathrm{p}}+\widetilde{W}_{\mathrm{p}} \\
& =W_{\mathrm{p}}\left(\frac{T_{\mathrm{p} 1}}{N_{\mathrm{p}}-T_{\mathrm{p} 2}}\right)
\end{aligned}
$$

it is easy to verify that

$$
W_{\mathrm{p}}=\left(\frac{N_{\mathrm{p}}-T_{\mathrm{p} 2}}{-N_{\mathrm{p}}+T_{\mathrm{p} 1}+T_{\mathrm{p} 2}}\right) \widetilde{W}_{\mathrm{p}}
$$

Using (13), equation (12) can be written as

$$
\bar{W}_{\mathrm{m}} r-y_{\mathrm{m}}^{*}=\left(\frac{N-T_{2}}{N} \frac{T_{\mathrm{p} 1}}{N_{\mathrm{p}}-T_{\mathrm{p} 2}}-\frac{T_{1}}{N}\right) W_{\mathrm{p}}\left(\theta^{* \mathrm{~T}} \omega\right)+\frac{T_{1}}{N}\left(\widetilde{W}_{\mathrm{m}} r-\widetilde{W}_{\mathrm{p}}\left(\theta^{* \mathrm{~T}} \omega\right)\right)
$$

and using (14), this finally becomes

$$
\bar{W}_{\mathrm{m}} r-y_{\mathrm{m}}^{*}=-\frac{T_{\mathrm{p} 1}}{N}\left(\frac{-N+T_{1}+T_{2}}{-N_{\mathrm{p}}+T_{\mathrm{p} 1}+T_{\mathrm{p} 2}}\right) \widetilde{W}_{\mathrm{p}}\left(\theta^{* \mathbf{T}} \omega\right)+\frac{T_{1}}{N} \widetilde{W}_{\mathrm{m}} r
$$

The meaning of (15) can be clarified as follows. Suppose that the unmodelled dynamics are exactly known and so the decomposition polynomials $T_{1}, T_{2}$ and $N$ can be chosen equal to $T_{\mathrm{p} 1}, T_{\mathrm{p} 2}$ and $N_{\mathrm{p}}$. Using (7), equation (15) then becomes

$$
\bar{W}_{\mathrm{m}} r-y_{\mathrm{m}}^{*}=\frac{T_{1}}{N}\left(W_{\mathrm{p}}\left(\theta^{* \mathrm{~T}}\left(\omega-\omega^{*}\right)\right)\right)
$$

Comparing (16) with (7), it is observed that the second part of the disturbance $\nu$ has vanished. Therefore a perfect decomposition removes the part from $\nu$ that corresponds to the unmodelled dynamics, which have as input the actual process input. The remaining disturbance consists of the nominal plant output with an input that depends directly on the error in the signal vector which is caused by the unmodelled dynamics, multiplied by $T_{1} / N$.

\subsection{The choice of the decomposition parameters}

While the linear part of the error equation is changed to $\left[\left(N-T_{2}\right) / N\right] \bar{W}_{\mathrm{m}}$, it is possible to obtain a more positive real error equation for increased stability. Because $\bar{W}_{\mathrm{m}}$ is unknown, the decomposition parameters cannot be chosen accurately to achieve this. In general, however, the poles determined by $N$ must have a proper damping ratio and the transfer $\left(N-T_{2}\right) / N$ should have a phase lead which is as large as possible in order to obtain the best results in this respect. Because the choice of $N$ is free, this can easily be achieved.

The second decomposition effect is a decrease in the output disturbance. To remove one of the terms in the disturbance completely, the model decomposition parameters should be as close as possible to the process parameters. While in a process decomposition also, $N_{\mathrm{p}}$ is free, this does not contradict the above-stated requirements on $N$. However, the remaining output disturbance term contains a factor $T_{1} / N$ and hence the choice of $N$ also affects this remaining 
disturbance. Therefore the best choice of $N$ may be a compromise between the two improvements of the error equation.

In addition to this, note that the decomposition changes the reference model output and hence the control goal. The analysis of the decomposition effects on the error equation, as presented above, does not disclose anything about the price to be paid in terms of performance. The freedom in choosing $N$ must therefore not only be used to find a decomposition model that makes the output error insensitive to the unmodelled dynamics, but must also result in a desired closed-loop response. Similarly, while an exact choice of $T_{1}$ and $T_{2}$ is favourable in terms of the error equation analysis, modifications of $T_{1}$ and $T_{2}$ may be necessary to achieve a proper closed-loop behaviour. Examples of this can be found below. To conclude, although the decomposition polynomials all have a clearly distinguishable function, the choice of the decomposition parameters is not trivial and depends on the problem at hand. Some feeling for the process is needed to obtain a proper tuning, which can best be obtained with the aid of simulation experiments.

\section{EXAMPLES}

This section presents two examples showing the decomposition design steps and the results obtained.

\subsection{A first example}

Process and model transfers and decomposition. The nominal process to be considered consists of only an integrator:

$$
W_{\mathrm{p}}=\frac{1}{s}
$$

In practice, however, there are extra first-order dynamics:

$$
\bar{W}_{\mathrm{p}}=\frac{1}{s\left(s \tau_{\mathrm{p}}+1\right)}
$$

Because the system is primarily designed for a process of order one, a first-order reference model is chosen:

$$
W_{\mathrm{m}}=\frac{1}{s+1}
$$

The primary controller needs two adjustable parameters $k_{0}$ and $d_{0}$ to calculate the control signal $u$ :

$$
u=k_{0} r+d_{0} y_{\mathrm{p}}
$$

The first-order reference model yields an SPR error equation in the nominal case and therefore an error-augmenting network is not needed. To compensate for the extra process dynamics, the decomposition is chosen to resemble a transfer $1 /\left(s \tau_{m}+1\right)$, yielding

$$
\frac{T_{1}}{N-T_{2}}=\frac{1}{S T_{\mathrm{m}}+1}
$$

Choosing the polynomial $N=s \tau_{\mathbf{n}}+1$ and inspecting this equation, it can be immediately seen 
that

$$
\begin{aligned}
T_{1} & =1 \\
N-T_{2} & =S \tau_{\mathrm{m}}+1 \Rightarrow T_{2}=\left(\tau_{\mathfrak{n}}-\tau_{\mathrm{m}}\right) s
\end{aligned}
$$

The parameter $\tau_{\mathrm{m}}$ represents the expected nominal time constant of the extra process dynamics. The design parameter $\tau_{\mathrm{n}}$ can be freely chosen and thereby determines $T_{2}$ and $N$. Note that in the implementation the pure differentiating terms in $T_{2}$ impose no problems because $y_{\mathrm{m}}$ is calculated as $y_{\mathrm{m}}=\left(T_{1} / N\right) W_{\mathrm{m}} r+\left(T_{2} / N\right) y_{\mathrm{p}}$.

Analysis of the error equation. For the nominal case, $k_{0}^{*}=1$ and $d_{0}^{*}=-1$. The actual system transfer function for these parameters is

$$
\frac{y_{\mathrm{p}}}{r}=\frac{1}{\tau_{\mathrm{p}} s^{2}+s+1}=\bar{W}_{\mathrm{m}}
$$

Assuming that the extra process dynamics are governed by $\tau_{p}=0 \cdot 5$, Figure 4 (plot ' $a$ ') shows that this actual error transfer is not SPR. If the decomposition method is applied, the error transfer changes to $\left[\left(N-T_{2}\right) / N\right] \bar{W}_{\mathrm{m}}$. Plots ' $\mathrm{b}$ ' and ' $\mathrm{c}$ ' in Figure 4 show the Nyquist plot of this modified transfer when $\tau_{\mathbf{n}}=0.1$ and 0.01 respectively. The decomposition moves the Nyquist plot to the first quadrant, making the real part of the error transfer positive over a larger frequency range.

The second decomposition effect lies in the modification of the output disturbance on the error equation. Evaluating equations (3) and (15), the original disturbance appears to be

$$
\nu=\frac{-\tau_{\mathrm{p}} s^{2}}{(s+1)\left(\tau_{\mathrm{p}} s^{2}+s+1\right)} r
$$

and the disturbance after decomposition, if $\tau_{\mathrm{p}}$ is exactly known, is

$$
\nu=\frac{\tau_{\mathrm{p}} s}{(s+1)\left(s \tau_{\mathrm{n}}+1\right)\left(\tau_{\mathrm{p}} s^{2}+s+1\right)} r
$$

If decomposition is used, the output disturbance depends on the design parameter $\tau_{\mathrm{n}}$. Figure 5 shows the norm of the disturbance in the original case (' $a$ ') as a function of the frequency. Plots ' $b$ '- ' $d$ ' show the disturbance magnitude for $\tau_{\mathbf{n}}=0 \cdot 1,1$ and 10 respectively. It is observed

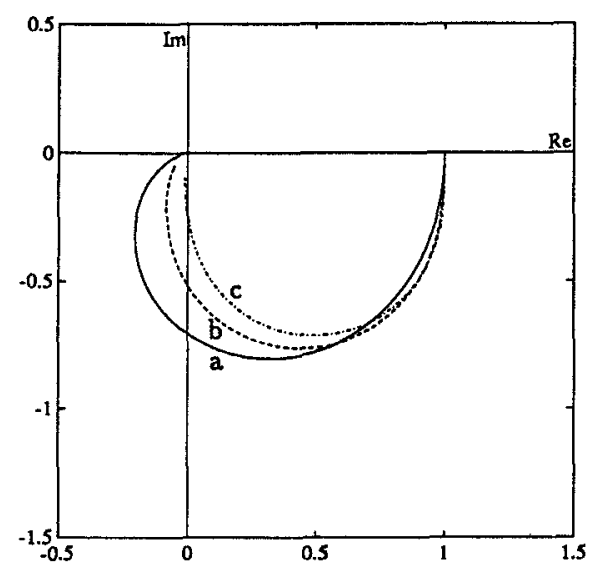

Figure 4. Example 1: Nyquist plots of $\bar{W}_{\mathrm{m}}$ and $\left[\left(N-T_{2}\right) / N\right] \bar{W}_{\mathrm{m}}-$ (a) original plot; (b) $\tau_{\mathrm{n}}=0 \cdot 1 ;$ (c) $\tau_{\mathrm{n}}=0 \cdot 01$ 


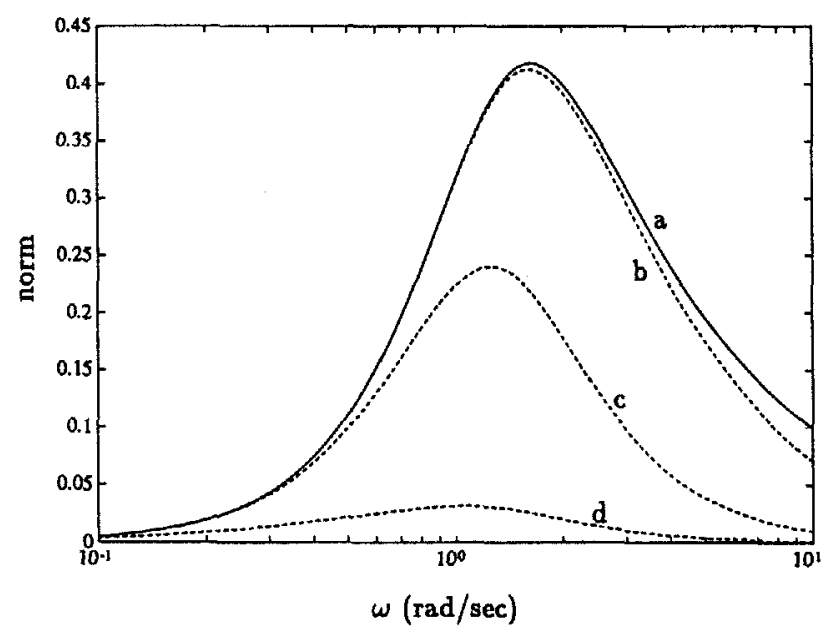

Figure 5. Disturbance magnitude as a function of frequency - (a) original disturbance $\left(\tau_{\mathrm{n}}=0\right)$; (b) $\tau_{\mathrm{n}}=0 \cdot 1$; (c) $\tau_{\mathrm{n}}=1$; (d) $\tau_{\mathrm{n}}=10$

that a large $\tau_{n}$ is favourable for a small disturbance magnitude. This contradicts the requirement on $\tau_{\mathrm{n}}$ for obtaining a more positive real error transfer, for which $\tau_{\mathrm{n}}$ should be as small as possible. Note that $\tau_{\mathrm{n}}$ can be chosen freely while still being able to implement an exact decomposition.

Simulation results. When no decomposition is applied, the result of Figure 6 is obtained if a block-type reference signal is applied. The lower-order controller cannot handle the extra dynamics and a proper convergence is not achieved. When the decomposition method is implemented and $\tau_{p}=0.5$ is exactly known, the response improves considerably, as shown in Figure 7. Comparing these figures, it is observed that the higher-order dynamics are much better handled if decomposition is used, at the cost of somewhat slower behaviour. Note also the considerably reduced control effort. In choosing $\tau_{\mathrm{n}}$, making the real part of the error

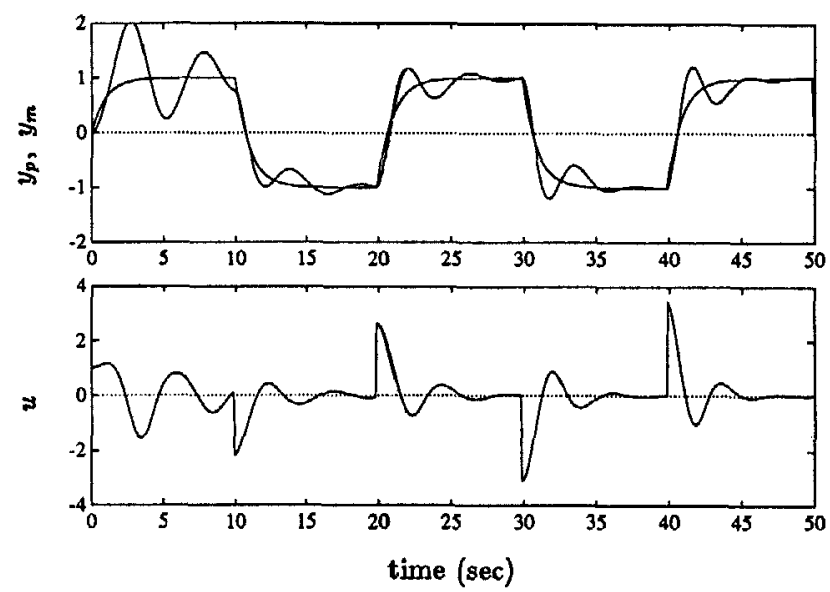

Figure 6. Example 1: result obtained without decomposition 


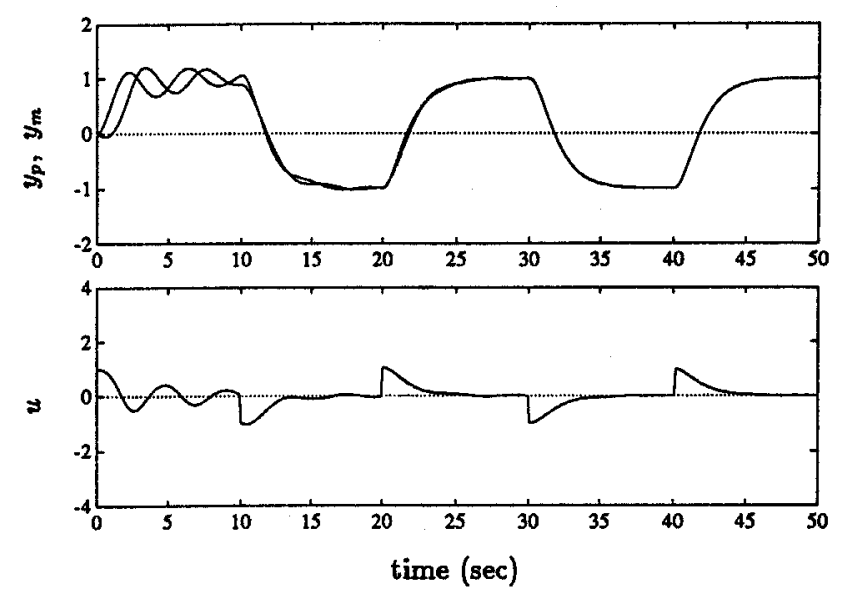

Figure 7. Example 1: result obtained by using exact decomposition
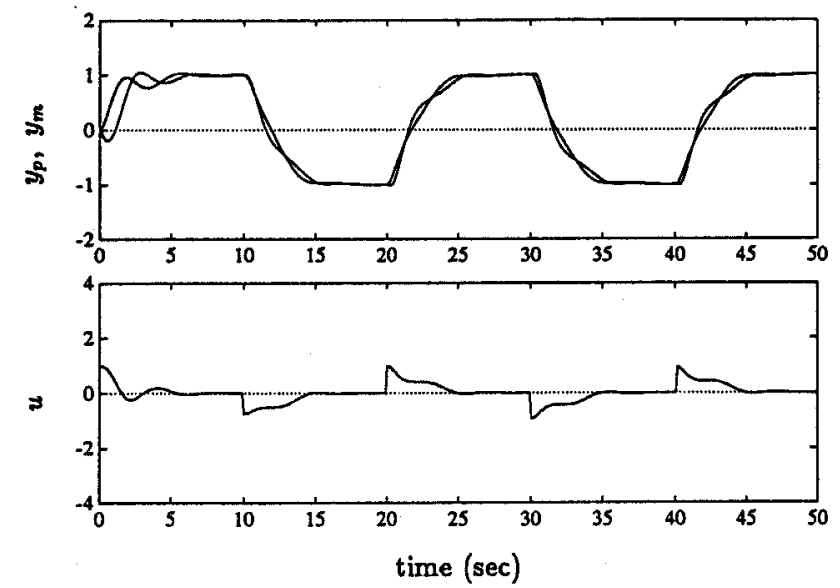

Figure 8. Example 1: result obtained by using non-nominal decomposition

equation more positive appears to have the largest effect, and $\tau_{\mathbf{n}}$ was chosen as $0 \cdot 25$. If the decomposition is no longer exact, for example if $\tau_{p}=0.25$ instead of 0.5 , the results are still satisfactory, as shown in Figure 8. This is due to the correction polynomial $T_{2}$.

\subsection{A second example}

Process and model transfers and decomposition. In this second example the nominal process considered has a transfer function

$$
W_{\mathrm{p}}=\frac{k_{\mathrm{p}}}{S \tau_{\mathrm{p}}+1}
$$

The real process, however, is in the form

$$
\bar{W}_{\mathrm{p}}=\frac{k_{\mathrm{p}}}{s \tau_{\mathrm{p}}+1}\left(\frac{\omega_{\mathrm{p}}^{2}}{s^{2}+2 z_{\mathrm{p}} \omega_{\mathrm{p}} s+\omega_{\mathrm{p}}^{2}}\right)
$$


The MRAC system is designed only for the 'modelled' part $k_{\mathrm{p}} /\left(s \tau_{\mathrm{p}}+1\right)$ and therefore is based on a system order of one. The reference model is chosen as

$$
W_{\mathrm{m}}=\frac{b}{s+a}
$$

For the decomposition a form is chosen which resembles the unmodelled part of the process:

$$
\frac{T_{1}}{N-T_{2}}=\frac{\omega_{\mathrm{m}}^{2}}{s^{2}+2 z_{\mathrm{m}} \omega_{\mathrm{m}} s+\omega_{\mathrm{m}}^{2}}
$$

Note that in this structure the decomposition polynomials $T_{1}$ and $N-T_{2}$ are determined by parameters $z_{\mathrm{m}}$ and $\omega_{\mathrm{m}}$ which represent design parameters that have an obvious meaning. If $z_{\mathrm{p}}$ is known to be small and hence the process poles are badly damped, it still appears to be useful to choose a proper decomposition model damping ratio $z_{\mathrm{m}}$ to obtain a non-oscillating model output. This illustrates well the borderline between what would be needed to obtain the best results in terms of improvements of the error equation, and what is actually desired. Choosing a low (but accurate) damping ratio $z_{m}$ decreases the sensitivity of the output error to the unmodelled dynamics but would result in oscillating behaviour of the reference model output. Hence an exact decomposition would not yield a desired response. However, in the case of the natural frequency $\omega_{\mathrm{m}}$ the situation is different. Imposing an arbitrary frequency on the oscillating system is not useful and would take much control effort. Therefore it is attempted to make the error equation insensitive to the oscillation frequency by choosing $\omega_{\mathrm{m}}$ equal to the nominal value of $\omega_{\mathrm{p}}$ (which of course may not be known accurately). The polynomial $N$ can be chosen arbitrarily as long as the order of $N$ equals at least two. Otherwise, the transfer $T_{2} / N$ has more zeros than poles, introducing implementation problems. Writing $N$ as

$$
N=s^{2}+2 z_{\mathrm{n}} \omega_{\mathrm{n}} s+\omega_{\mathrm{n}}^{2}
$$

it is found that

$$
T_{2}=2\left(z_{\mathrm{n}} \omega_{\mathrm{n}}-z_{\mathrm{m}} \omega_{\mathrm{m}}\right) s+\left(\omega_{\mathrm{n}}^{2}-\omega_{\mathrm{m}}^{2}\right)
$$

Inspecting (19), it is observed that if $\omega_{\mathrm{n}}=\omega_{\mathrm{m}}$ and $z_{\mathrm{n}}=z_{\mathrm{m}}, T_{2}$ vanishes and no correction to $y_{\mathrm{m}}$ is made. In that case the knowledge of the unmodelled dynamics is only incorporated in the reference model. Recalling that the error equation profits from a phase lead correction to achieve a more positive real part, $1 / N$ can best be chosen relatively fast in this respect. $\omega_{n}$ is therefore chosen larger than $\omega_{\mathrm{m}}$. The damping ratio is chosen as $z_{\mathrm{n}}=1$.

Analysis of the error equation. This paragraph analyses the effects that the decomposition has on the error equation in this example. To test the decomposition method, the following process parameters are assumed: $k_{\mathrm{p}}=0.5, \tau_{\mathrm{p}}=1, \omega_{\mathrm{p}}^{2}=4$ and $z_{\mathrm{p}}=0.7$. The reference model parameters are $a=4$ and $b=4$. The same primary controller as in the first example is used:

$$
u=k_{0} r+d_{0} y_{\mathrm{p}}
$$

Because the nominal process is of order one, no ASGs are needed and an error-augmenting network is not required. For the undisturbed process $W_{\mathrm{p}}$, parameter values $k_{0}^{*}=8$ and $d_{0}^{*}=-6$ make $W_{\mathrm{p}}\left(\theta^{* \mathrm{~T}} \omega^{*}\right)$ equal to $W_{\mathrm{m}} r$. In the disturbed case the linear part of the error equation is

$$
\bar{W}_{\mathrm{m}}=\frac{16}{s^{3}+3 \cdot 8 s^{2}+6 \cdot 8 s+16}
$$




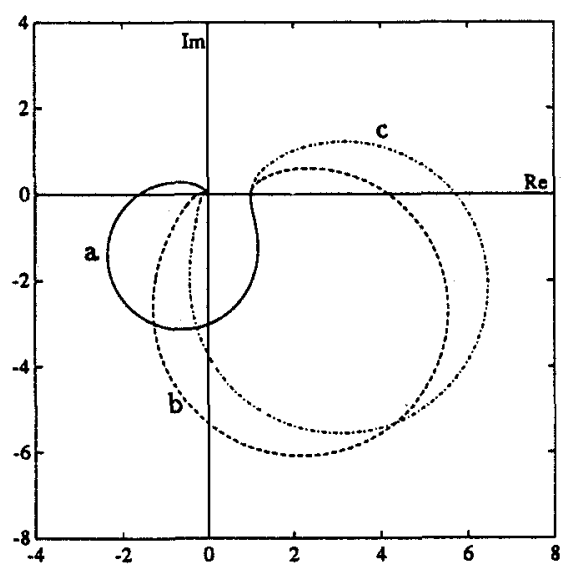

Figure 9. Example 2: Nyquist plots of $\bar{W}_{\mathrm{m}}$ and $\left[\left(N-T_{2}\right) / N\right] \bar{W}_{\mathrm{m}}-$ (a) original plot; (b) $N=s^{2}+20 s+100$; (c) $N=s^{2}+63 s+1000$

Figure 9 shows the Nyquist plot of $\bar{W}_{\mathrm{m}}$ (denoted by 'a'), which lies mainly in the second quadrant. Also in Figure 9, Nyquist plots of $\left[\left(N-T_{2}\right) / N\right] \bar{W}_{\mathrm{m}}$ are shown with $N-T_{2}=s^{2}+4 s+4, \quad N=s^{2}+20 s+100$ and $N=s^{2}+63 s+1000$ (plots 'b' and 'c' respectively). To be able to compare the position of these plots in the complex plane, plots ' $b$ ' and ' $c$ ' are corrected for their lower DC gain. Obviously, making $1 / N$ faster has the effect of $\left[\left(N-T_{2}\right) / N\right] \bar{W}_{\mathrm{m}}$ moving to the first quadrant, increasing the range over which the real part of the error equation's frequency response is positive.

For the disturbance on the output error an analysis similar to that in the first example can be worked out. In this case also, a small $\omega_{n}$ decreases the disturbance most. A proper compromise value for $\omega_{n}$ must be found such that the overall result is the best.

Simulation results. If no decomposition is used, the unmodelled dynamics induce unstable behaviour, as seen in Figure 10. Here the nominal process parameter values are assumed. If the nominal value of $\omega_{\mathrm{p}}^{2}$ is known, application of decomposition with $z_{\mathrm{m}}=1$ and $\omega_{\mathrm{m}}=\omega_{\mathrm{p}}$ gives

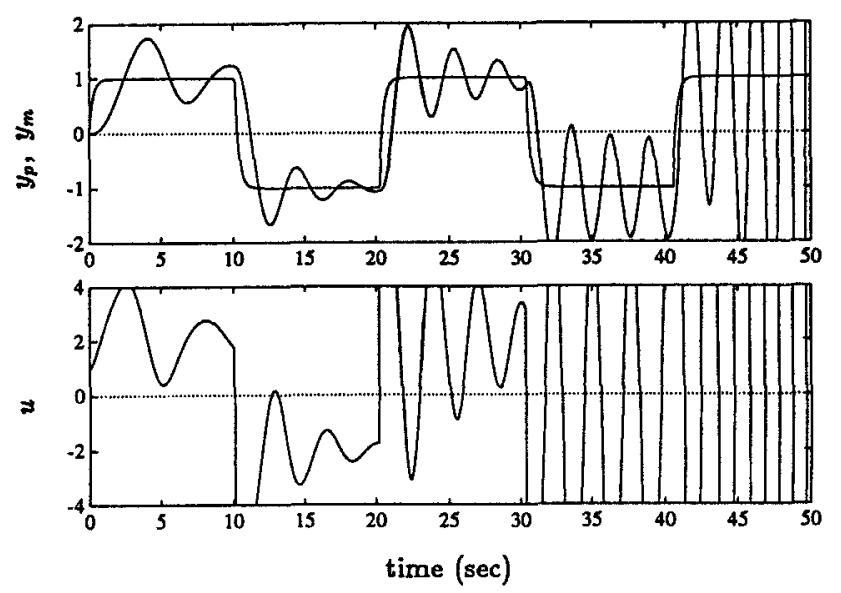

Figure 10. Example 2: result obtained without decomposition 

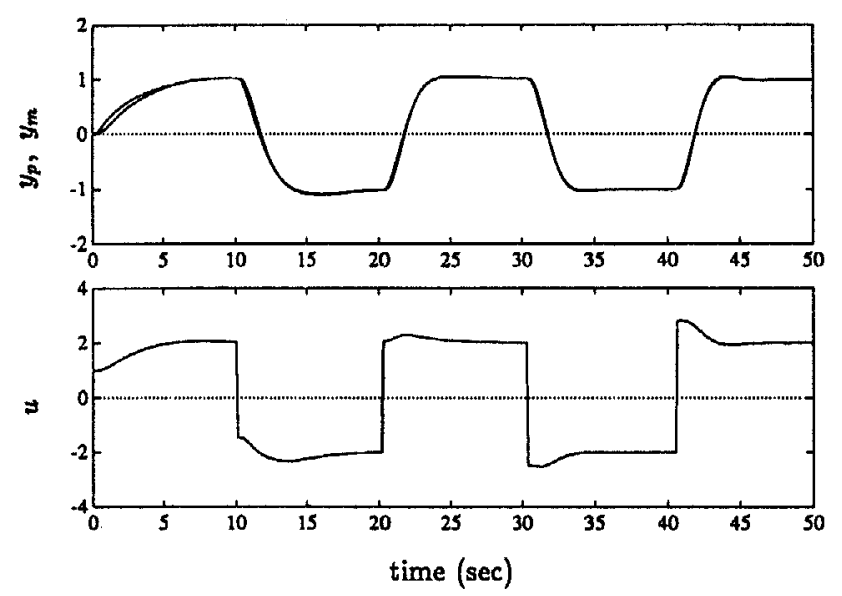

Figure 11. Example 2: result obtained with nominal process if the decomposition parameters are known exactly
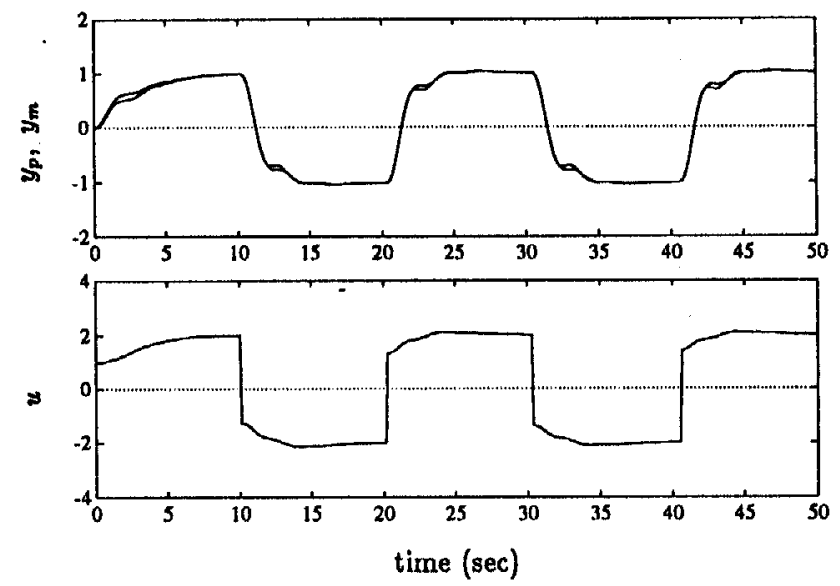

Figure 12. Example 2: result obtained with non-nominal process

the result shown in Figure 11. Here the polynomial $N=s^{2}+8 s+16$ is found to be an acceptable compromise between the two requirements. If the parameters of the structured unmodelled dynamics are not known exactly, a less exact decomposition arises. To test the robustness of the system with respect to mismatch between process and decomposition parameters, Figure 12 shows the results with $\omega_{p}^{2}=8$ and $z_{p}=0.25$. It is seen that the response is still satisfactory owing to the correction by $T_{2}$.

It should be noted that when using a full-order controller for the process including unmodelled dynamics, a better response than that shown in Figure 10 can be obtained. However, a total number of six parameters would need to be adjusted and so the controller is much more complex. In a practical application an as-small-as-possible number of adjustable parameters is favoured. In addition, a full-order controller would require a much larger control effort. A practical example in which these aspects are very apparent is presented in Reference 22, where the decomposition method is applied to a scale model of a gantry crane.

Comparing the responses with decomposition and those without, it is observed that the 
decomposition method improves the adaptive system behaviour if unmodelled dynamics with a known structure are present. This improvement is achieved through adjustment of the reference model output, and comparing the original $y_{\mathrm{m}}$ in Figure 10 with the adjusted $y_{\mathrm{m}}$ in Figure 11 shows a somewhat slower response because of the decomposition. Hence the improvement is achieved by adjusting the control goal to compensate for a lower controller order than necessary for perfect model matching.

\section{DECOMPOSITION DESIGN STEPS}

This section summarizes the design steps that have to be taken when applying the decomposition method.

1. The first step in the design is the decision as to which part of the process dynamics is to be regarded as 'nominal' and which part is considered 'unmodelled'. The controller order can then be chosen such that the nominal part can be controlled properly and the controller design can take place. The decision can be based on the desired system specifications and the feasibility of a higher-order controller.

2. In the next step the structure of the 'unmodelled' process dynamics is analysed and copied into the decomposition model. In this model the parameters are chosen as well as possible to resemble the actual process parameters. However, the response of the decomposition model is modified such that it represents an acceptable output behaviour. For example, to an oscillatory part a damping ratio is added.

3. The modified structure is finally transformed into polynomials $T_{1}$ and $N-T_{2}$. In the decomposition structure the polynomial $N$ can be chosen freely (therewith determining $T_{2}$ ). As shown above, the two decomposition effects impose different requirements on the choice of these polynomials. In addition, the resulting closed-loop behaviour depends on $N$ and hence $N$ influences the performance of the system. A satisfactory polynomial $N$ for a specific situation can best be determined in simulation.

\section{CONCLUSIONS}

The primary goal of reference model decomposition is improved system behaviour if there are unmodelled process dynamics with a known structure. Alternatively, decomposition can be regarded as a method that allows the use of a lower-order controller than is required for perfect model matching. In particular, the latter property is useful in a practical application, in which the requirement that rough knowledge about the unmodelled dynamics structure and parameters must be present is generally satisfied. Using a lower-order adaptive controller without decomposition, the adaptive system tries to impose the reference model response on the process without taking into consideration the limited model-matching capability of the primary controller. This may easily result in instability.

Usually, structural knowledge of the plant dynamics exists in the low-frequency area, whereas uncertainty is mainly present in the high-frequency range. Therefore high-frequency unmodelled dynamics cannot be coped with by the decomposition method and adaptive law modifications should be used. The plant dynamics are then divided into three classes: 'nominal', 'structured unmodelled' and 'unmodelled'. The primary controller is equipped for the nominal part. Reference model decomposition makes the adaptive system robust with respect to the structured unmodelled part. The real unmodelled dynamics (usually of high frequency) can be dealt with by standard modifications of the adaptive law, which guarantee stability if the unmodelled dynamics play a minor role in the process output. 
The decomposition method can be regarded as a compromise between perfect model matching and stability. The reference model is adjusted to the process such that its output takes the actually achievable process response into account. This adjustment is of a direct form. An adaptive version of reference model adjustment is presented in Reference 16. Because the reference model output is adjusted for the unmodelled dynamics, these dynamics have less influence on the output error and thus on the parameter adaptation. The model adjustment results in a somewhat slower reference model output. However, the stability properties are improved considerably and, as a side effect, the control effort is reduced.

The decomposition has two effects on the error equation of the adaptive system. First, the real part of the error equation's frequency response is made positive over a wider frequency range. Secondly, the output disturbance on the error equation is reduced. These two effects result in improved system properties in the sense of existing robustness analysis methods. However, it should be stressed that although it can be felt intuitively that the stability properties are improved, no theoretic stability guarantee has been given. In addition, no link has yet been laid between the choice of the decomposition parameters and the resulting closedloop behaviour, and, for example, the maximum deviation of the decomposition parameters from the actual process parameters has not yet been established. Despite this lack of a solid mathematical basis, simulation experiments and a practical application all showed the usefulness of the method.

\section{REFERENCES}

1. Ioannou, P. A., and K. S. Tsakalis, 'A robust direct adaptive controller', IEEE Trans. Automatic Control, AC-31, 1033-1043 (1986).

2. Ioannou, P. A., and G. Tao, 'Dominant richness and improvement of performance of robust adaptive control', Automatica, 25, 287-291 (1989).

3. Kosut, R. L., 'Methods of averaging for adaptive systems', in Adaptive and Learning Systems: Theory and Applications, Plenum, New York, 1986, pp. 33-45.

4. Kreisselmeier, G., and B. D. O. Anderson, 'Robust model reference adaptive control', IEEE Trans. Automatic Control, AC-31, 127-133 (1986).

5. Lee, T. H., and K. S. Narendra, 'Robust adaptive control of discrete-time systems using persistent excitation', Automatica, 24, 781-788 (1988).

6. Narendra, K. S., and A. M. Annaswamy, 'Robust adaptive control', in Adaptive and Learning Systems: Theory and Applications, Plenum, New York, 1986, pp. 3-31.

7. Narendra, K. S., and A. M. Annaswamy, 'Robust adaptive control in the presence of bounded disturbances', IEEE Trans. Automatic Control, AC-31, 306-315 (1986).

8. Peterson, B. P., and K. S. Narendra, 'Bounded error adaptive control', IEEE Trans. Automatic Control, AC-27, 1161-1168 (1982).

9. Kreisselmeier, G., and K. S. Narendra, 'Stable model reference adaptive control in the presence of bounded disturbances', IEEE Trans. Automatic Control, AC-27, 1169-1175 (1982).

10. Sung, D. J. T., and T. T. Lee, 'Model reference adaptive control of a solenoid valve controlled hydraulic system', Int. J. Syst. Sci., 18, 2065-2091 (1987).

11. Horowitz, R., M. C. Tsai, G. Anwar, and M. Tomizuka, 'Model reference adaptive control of a two axis direct drive manipulator arm', Proc. IEEE Int. Conf. on Robotics and Automation, 1987, pp. 1216-1222.

12. Billerbeck, G., and L. Klinsmann, 'Micro-processor implementation of a model reference adaptive controller in view of wide industrial applicability', Syst. Anal., Modell. Simul., 4, 259 262 (1987).

13. Kuo, C. Y., and W. Worger, 'Discrete time model reference adaptive control of an industrial manipulator's waist joint', Trans. ASME, 109, 294-297 (1987).

14. Begovich, O., and R. Ortega, 'Adaptive head control of a hydraulic open channel model', Automatica, 25, $103-107$ (1989).

15. Dumont, G. A., M. Martin-Sanchez, and C. C. Zervos, 'Comparison of an auto-tuned PID regulator and an adaptive predictive control system on an industrial bleach plant', Automatica, 25, 33-40 (1989).

16. Butler, H., G. Honderd, and J. van Amerongen, 'Model reference adaptive control of a direct-drive DC motor', IEEE Control Syst. Mag., 9, 80-84 (1989).

17. Narendra, K. S., and A. M. Annaswamy, Stable Adaptive Systems, Prentice-Hall, Englewood Cliffs, NJ (1989).

18. Rohrs, C. E., L. Valavani, M. Athans, and G. Stein, 'Robustness of continuous-time adaptive control algorithms in the presence of unmodelled dynamics', IEEE Trans. Automatic Control, AC-30, 881-889 (1985). 
19. Àström, K. J., 'Analysis of Rohrs counterexamples to adaptive control', Proc. 22nd IEEE Conf. on Decision and Control, San Antonio, TX, 1983, pp. 982-987.

20. Sastry, S. S., and M. Bodson, Adaptive Control - Stability, Convergence, and Robustness, Prentice-Hall, Englewood Cliffs, NJ (1989).

21. Van Den Bosch, P. P. J., 'Model reference adaptive control: model updating', in Systems and Control Encyclopedia, Pergamon, Oxford, 1987, pp. 3070-3071.

22. Butler, H., G. Honderd, and J, van Amerongen, 'Model reference adaptive control of a gantry crane scale model', IEEE Control Syst. Mag., 11, 57-62 (1991). 